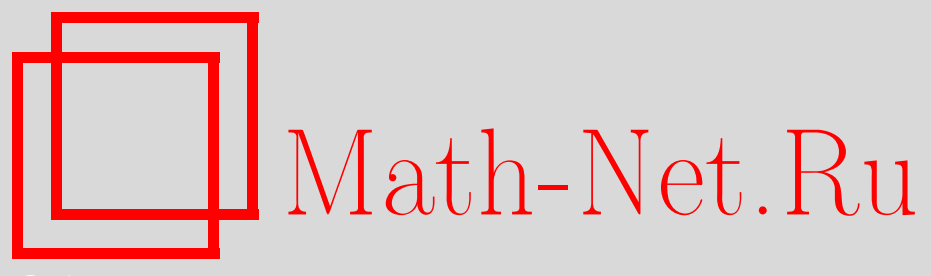

Э. Б. Винберг, С. Е. Кузнецов, Евгений Борисович Дынкин (некролог), УМН, 2016, том 71, выпуск 2, 179-204

DOI: https://doi.org/10.4213/rm9715

Использование Общероссийского математического портала Math-Net.Ru подразумевает, что вы прочитали и согласны с пользовательским соглашением http://www . mathnet.ru/rus/agreement

Параметры загрузки:

IP : 18.208 .226 .222

26 апреля 2023 г., 11:50:22

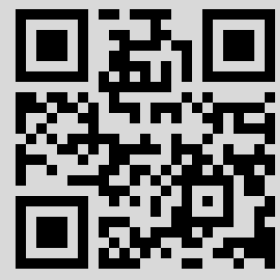




\section{Евгений Борисович Дынкин}

14 ноября 2014 г. на 91-м году жизни скончался выдающийся математик Евгений Борисович Дынкин, преподававший на механико-математическом факультете МГУ в 1948-1968 гг., a c 1977 г. являвшийся профессором Корнельского университета (США). Мировую известность получили его работы в двух разных областях математики: теории групп Ли и теории вероятностей.

Евгений Борисович родился в Ленинграде 11 мая 1924 г. Отец его был юристом, мать - зубным врачом, оба родом из Белоруссии. В 1935 г., вскоре после убийства Кирова, семью отправили в административную ссылку в Казахстан. В 1937 г. отца арестовали, и он исчез в Гулаге. В 1940 г. Женя Дынкин с блеском окончил школу в Актюбинске, перепрыгнув через класс, и поступил на механико-математический факультет МГУ.

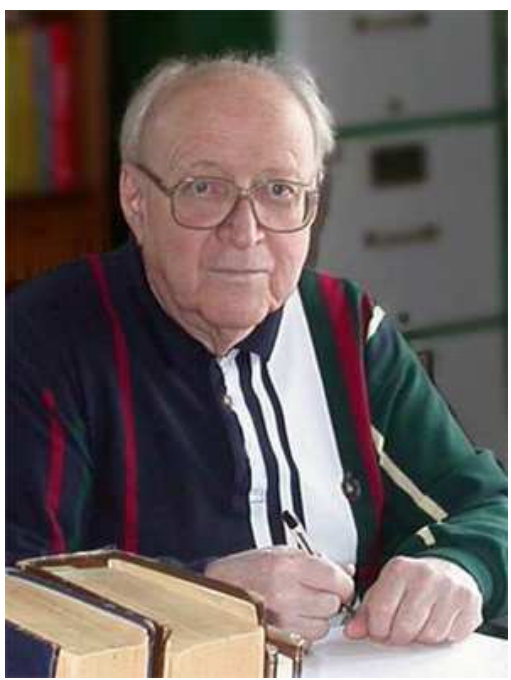

После начала войны Женя уехал в Тульскую область, где в то время работала его мать по окончании срока ссылки, а затем они эвакуировались в Пермь. В армию его не призывали по причине сильной близорукости и перенесенного в детстве костного туберкулеза. Он продолжил обучение в Пермском университете, где проучился до конца 1943 г. Большое участие в его жизни приняла С. А. Яновская, профессор мехмата МГУ, оказавшаяся в то время в Перми. Она порекомендовала его И. М. Гельфанду, и по возвращении в Москву Гельфанд пригласил его участвовать в своем семинаре.

Будучи студентом четвертого курса, Женя Дынкин по поручению Гельфанда готовил доклад о структуре и классификации полупростых алгебр Ли по работам Картана, Вейля и Ван-дер-Вардена. В процессе подготовки он придумал "системы простых корней", позволившие кардинально упростить классификацию и впоследствии полностью изменившие облик теории полупростых алгебр Ли. Это была его первая (по времени выполнения) научная работа.

На пятом курсе Женя Дынкин работал в семинаре А.Н. Колмогорова и вместе с другим участником семинара Колей Дмитриевым решил предложенную Колмогоровым задачу о характеристических числах стохастической матрицы. Это была его вторая сданная в печать работа.

По окончании университета в 1945 г. он поступил в аспирантуру, где его руководителем был Колмогоров. Евгений Борисович досрочно защитил кандидатскую диссертацию и в 1948 г. был зачислен старшим преподавателем на кафедру теории вероятностей мехмата МГУ. Еще через год Евгений Борисович стал доцентом, а в 1951 г. защитил докторскую диссертацию и вскоре стал профессором. Обе диссертации были 
посвящены группам и алгебрам Ли. За эти работы, ставшие впоследствии классическими, в 1951 г. ему была присуждена премия Московского математического общества.

Начиная с 1955 г. Е.Б. Дынкин полностью переключается на теорию вероятностей. Его работы в этой области в основном посвящены теории марковских процессов, одним из основателей которой он может считаться. Его фундаментальная монография "Марковские процессы" [17], появившаяся в 1963 г., в течение многих лет служила стандартным источником информации в этой области и продолжает оставаться классическим изложением теории. Особо стоит отметить последний цикл работ Е. Б. Дынкина; посвященный суперпроцессам - марковским процессам, связанным с нелинейными уравнениями в частных производных. Менее известны широкому кругу математиков работы Евгения Борисовича по теории игр и математической экономике, выполненные в 1960-1970-х годах. Их значение стало раскрываться только в последние годы, особенно в связи с недавно открытыми применениями "игр Дынкина" в финансовой математике.

Е. Б. Дынкин был блестящим лектором и замечательным педагогом. Будучи профессором кафедры теории вероятностей, он читал лекции и по алгебре, и по анализу. Его лекции и семинары никогда не были скучными. Он умел заинтересовать слушателей неординарным изложением материала и постановкой интересных задач, и у него всегда было много учеников. Созданный им семинар по группам Ли работает до сих пор под руководством его учеников Э. Б. Винберга и А. Л. Онищика, которые создали свои собственные научные школы. Участниками семинара Дынкина по группам Ли в разное время были также В. И. Арнольд, Ф. А. Березин, Ф. И. Карпелевич, И. И. Шапиро-Пятецкий и другие, ставшие впоследствии известными математиками. Среди учеников Е.Б. Дынкина по теории вероятностей - многие лидеры следующего поколения: Н. В. Крылов, М.Б. Малютов, С. А. Молчанов, А. В. Скороход, М.И. Фрейдлин, Р.З. Хасьминский и др. Значительное число математиков, включая нескольких, ставших позднее знаменитыми, связывают свои первые научные результаты именно с Е.Б. Дынкиным, даже если он не был их формальным руководителем.

Еще обучаясь в Пермском университете, Евгений Борисович организовал кружок для трех других студентов МГУ, также оказавшихся в Перми. Вернувшись в Москву, он стал руководителем одной из секций математического кружка для школьников. По материалам работы этой секции в 1945-1947 гг. им вместе с участником этого кружка В.А. Успенским была впоследствии написана книга "Математические беседы", ставшая классической в жанре популярной образовательной литературы.

В начале 1960-х годов, в связи с проводившейся тогда реформой среднего образования, в СССР появились специальные школы с математическим уклоном. Евгений Борисович сыграл выдающуюся роль в их ранней истории. В 1963 г. он с помощью своих аспирантов и других молодых математиков организовал Вечернюю математическую школу (ВМШ) при школе № 2 г. Москвы, а на следующий год, при содействии директора школы В. Ф. Овчинникова, организовал в ней же поток для математически одаренных школьников 9-10 классов (1964-1966 гг.). Здесь в полной мере проявился организационный талант Евгения Борисовича. С помощью продуманной системы лекций и практических занятий, конкурсов по решению задач была создана неповторимая атмосфера соревнования.

Определенная независимость во взглядах Е.Б. Дынкина не устраивала власти, и весной 1968 г. ему пришлось уйти из МГУ. Пробыв какое-то время без работы, он в конце концов стал научным сотрудником отдела математики ЦЭМИ (Центрального

${ }^{1}$ Е. Б. Дынкин, В.А. Успенский, Математические беседъ. Задачи о многоцветной раскраске. Задачи из теории чисел. Случайные блуждания, Библ. матем. кружка, 6, Гостехиздат, М.-Л., 1952, 288 с. - Прим. ред. 
экономико-математического института) АН СССР. Даже в это время он организует у себя дома семинар для узкого круга студентов.

В 1976 г. Евгений Борисович решился на эмиграцию. Он принял предложение Корнельского университета (Итака, США) и получил там почетную именную позицию. Евгений Борисович проработал в Корнельском университете 33 года и вышел в отставку лишь после того, как ему исполнилось 86 лет. Вплоть до последнего времени он поддерживал тесные связи со своими российскими учениками и коллегами.

В течение нескольких десятилетий Е. Б. Дынкин брал интервью у известных российских и иностранных математиков. Основным делом последних лет его жизни стала систематизация этой обширной коллекции аудио- и видеозаписей и перевод их в письменную форму, а также перевод на английский язык русскоязычных интервью. Эта коллекция доступна теперь на сайте Корнельского университета ${ }^{2}$.

В 1985 г. Е.Б. Дынкин был избран членом Национальной академии наук США. В 1995 г. Московское математическое общество, вице-президентом которого Е. Б. Дынкин был в 1964-1971 гг., избрало его почетным членом. Он также был почетным доктором нескольких университетов, в том числе Независимого московского университета.

Е. Б. Дынкин - автор более 200 научных работ, восьми научных монографий и трех популярных образовательных книг. До самого последнего времени он вел активную научную работу. Последняя из его работ была опубликована в 2013 г. В 2000 г. Американское математическое общество выпустило том его избранных работ с комментариями специалистов [51].

В настоящей статье мы даем обзор работ Дынкина по теории групп Ли и по теории вероятностей. О его работах по математической экономике можно прочитать в обзоре И. В. Евстигнеева в [51]. В список литературы включены только те работы Дынкина, на которые имеются ссылки в тексте. Это, как правило, не первые краткие сообщения о результатах, а более поздние статьи, содержащие полные доказательства.

\section{1. Работы Е. Б. Дынкина по группам Ли}

1.1. Системы простых корней. Как известно, классификация комплексных полупростых алгебр Ли, полученная в конце XIX в. В. Киллингом и Э. Картаном, основана на рассмотрении весового разложения алгебры Ли относительно ее картановской подалгебры. Таким образом по каждой полупростой алгебре Ли $\mathfrak{g}$ строится система корней $\Delta$ - конечная система ненулевых векторов евклидова пространства $E$ (размерность которого равна рангу алгебры $\mathfrak{g}$ ), обладающая следующими свойствами:

(К0) система $\Delta$ порождает пространство $E$;

(K1) если $\alpha \in \Delta$, то $k \alpha \in \Delta$ тогда и только тогда, когда $k= \pm 1$;

(К2) если $\alpha, \beta \in \Delta$, то $(\alpha \mid \beta)=: 2(\alpha, \beta) /(\alpha, \alpha) \in \mathbb{Z}$;

(К3) для любого $\alpha \in \Delta$ система $\Delta$ переходит в себя при отражении

$$
R_{\alpha}: x \mapsto x-(\alpha \mid x) \alpha
$$

Группа $W$, порожденная отражениями $R_{\alpha}$, называется группой Вейля системы $\Delta$ (или алгебры $\mathfrak{g}$ ). Смысл условия (K2) состоит в том, что группа $W$ сохраняет решетку, порожденную корнями.

Алгебра g однозначно определяется своей системой корней, и всякая система векторов евклидова пространства, удовлетворяющая условиям (K0)-(K3), является системой корней некоторой полупростой алгебры Ли.

\footnotetext{
${ }^{2}$ http://dynkincollection.library.cornell.edu.
} 
Прямой суммой систем корней называется их объединение, рассматриваемое как система векторов в прямой сумме евклидовых пространств. Система корней называется неразложимой, если она не может быть представлена в виде прямой суммы двух систем корней. Неразложимые системы корней отвечают простым алгебрам Ли. Система корней простой алгебры Ли определена однозначно с точностью до гомотетии.

До работ Е.Б. Дынкина все системы корней были известны, но не было единообразного способа их построения. Идея Е. Б. Дынкина [2] состояла в замене системы корней более простым объектом, однозначно ее определяющим. А именно, он предложил разбить корни на положительные и (противоположные им) отрицательные в смысле лексикографического упорядочения, определяемого каким-либо базисом пространства $E$, и рассмотреть положительные корни, которые не могут быть представлены в виде суммы двух положительных корней. Он назвал эти корни простыми и доказал, что система П простых корней составляет базис пространства $E$ и обладает следующим свойством:

(П) $(\alpha \mid \beta) \in \mathbb{Z}_{-}$для любых различных корней $\alpha, \beta \in \Pi$.

Система простых корней с точностью до действия группы Вейля не зависит от выбора базиса, относительно которого определяется лексикографическое упорядочение, и по ней однозначно восстанавливается система корней $\Delta$. Более того, всякий базис П пространства $E$, обладающий свойством (П), является системой простых корней некоторой системы корней $\Delta ;$ система $\Delta$ неразложима тогда и только тогда, когда система П неразложима. Тем самым классификация систем корней (а значит, и полупростых комплексных алгебр Ли) сводится к несложной задаче линейной алгебры.

Из условия (П) следует, что угол между двумя простыми корнями имеет вид $\pi-\pi / m$, где $m \in\{2,3,4,6\}$, а отношение квадратов их длин равно 1,2 или 3 , если $m=3,4$ или 6 соответственно. Е. Б. Дынкин предложил описание системы простых корней при помощи графа, в котором вершины соответствуют простым корням и две вершины, соответствующие корням $\alpha$ и $\beta$, соединены простым, двойным или тройным ребром, если $m=3,4$ или 6 соответственно; кроме того, в случае наличия корней разной длины в одной связной компоненте вершины, соответствующие коротким корням, закрашиваются черным цветом. (Впоследствии вместо раскрашивания вершин стали ориентировать кратные ребра.) Эти графы, названные схемами Дънкина, в сущности являются модификацией схем Кокстера, классифицировавшего конечные линейные группы, порожденные отражениями, вне связи с системами корней. Неразложимость системы корней равносильна связности ее схемы Дынкина.

Без преувеличения можно сказать, что системы простых корней (и описывающие их схемы Дынкина) произвели революцию в теории полупростых групп и алгебр Ли. Не только классификация самих полупростых комплексных алгебр Ли, но и описание их линейных представлений, автоморфизмов, подалгебр, вещественных форм и прочих связанных с ними объектов естественно формулируются на языке простых корней. Так, неприводимое линейное представление полупростой алгебры Ли определяется своим старшим весом $\Lambda$, который, в свою очередь, задается своими "числовыми отметками" $(\alpha \mid \Lambda)$ на схеме Дынкина, причем этими отметками могут быть любые неотрицательные целые числа. Группа внешних автоморфизмов полупростой алгебры Ли естественно изоморфна группе автоморфизмов ее схемы Дынкина [5]. Классы сопряженных параболических подалгебр (подалгебр, содержащих максимальную разрешимую подалгебру) находятся в естественной биекции с подмножествами системы простых корней [76].

1.2. Полупростые подалгебры полупростых алгебр Ли. В 1950-1952 гг. в связи с проблемой С. Ли о классификации примитивных непрерывных групп преобразований Е. Б. Дынкин выполнил огромную программу классификации максимальных связных подгрупп Ли полупростых комплексных групп Ли или, что то же, клас- 
сификации максимальных подалгебр полупростых комплексных алгебр Ли [7], [8]. Эти результаты составили его докторскую диссертацию.

Еще В. В. Морозов в своей докторской диссертации 1943 г. [87] доказал, что всякая неполупростая максимальная подалгебра полупростой алгебры Ли является параболической подалгеброй, и тем самым свел классификацию максимальных подалгебр полупростых алгебр Ли к классификации полупростых максимальных подалгебр. С другой стороны, исследование полупростых подалгебр произвольных полупростых алгебр Ли легко сводится к исследованию полупростых подалгебр простых алгебр Ли.

Для классических простых комплексных групп Ли $G=\mathrm{SL}(V), \operatorname{SO}(V), \operatorname{Sp}(V)$ (где $V$ - комплексное векторное пространство) ответ, полученный Е.Б. Дынкиным [7], выглядит следующим образом.

Пусть $H \subset G$ - полупростая максимальная связная подгруппа Ли группы $G$. Если $H$ - приводимая линейная группа, то $G$ есть $\mathrm{SO}(V)$ или $\operatorname{Sp}(V)$, а $H$ есть связная компонента стабилизатора некоторого невырожденного подпространства $U \subset V$. Если группа $H$ неприводима, но не проста, то она является тензорным произведением двух классических линейных групп, а именно, $H=\mathrm{SL}(U) \otimes \mathrm{SL}(W), \mathrm{SO}(U) \otimes$ $\mathrm{SO}(W), \mathrm{Sp}(U) \otimes \mathrm{Sp}(W)$ или $\mathrm{SO}(U) \otimes \mathrm{Sp}(W)$, где $U \otimes W=V$.

В наиболее интересном случае, когда группа $H$ проста и неприводима, Е. Б. Дынкин получил следующий удивительный результат. Он доказал, что неприводимая простая связная линейная группа $H \subset \mathrm{SL}(V)$, как правило, является максимальной связной подгруппой Ли либо в $\mathrm{SL}(V)$, либо - если она сохраняет невырожденную билинейную форму - в $\mathrm{SO}(V)$ или $\mathrm{Sp}(V)$. Полный список исключений из этого правила содержит 4 серии и 14 отдельных линейных групп. Для всех этих случаев в [7] явно указана связная простая линейная группа, строго содержащая $H$ и отличная от соответствующей классической линейной группы.

По ходу дела в работе [7] были доказаны некоторые общие теоремы о линейных представлениях полупростых алгебр Ли, имеющие самостоятельный интерес. В частности, там были найдены некоторые неприводимые компоненты произведения неприводимых представлений полупростой алгебры Ли со старшими весами $\Lambda$ и $M$, отличные от старшей (картановской) компоненты, старший вес которой равен $\Lambda+M$. А именно, пусть $H(\Lambda, M)$ - множество старших весов всех неприводимых компонент указанного произведения. Введем частичное упорядочение на группе весов, полагая $\lambda \geqslant \mu$, если $\lambda-\mu$ есть линейная комбинация простых корней с целыми неотрицательными коэффициентами. Тогда $\Lambda+M$ является единственным максимальным элементом множества $H(\Lambda, M)$. В работе [7] были найдены все максимальные элементы множества $H(\Lambda, M) \backslash\{\Lambda+M\}$. Оказывается, что это в точности веса вида

$$
\Lambda+M-\alpha_{1}-\cdots-\alpha_{k},
$$

где $\alpha_{1}, \ldots, \alpha_{k}$ - различные простые корни, такие, что в последовательности $\Lambda, \alpha_{1}, \ldots$, $\alpha_{k}, M$ любые соседние члены не ортогональны, а не соседние, за исключением, быть может, пары $(\Lambda, M),-$ ортогональны. Соответствующие неприводимые компоненты входят в разложение с кратностью единица (как и компонента со старшим весом $\Lambda+M)$, и их старшие векторы предъявляются.

В следующей работе [8] Е. Б. Дынкин перечисляет все полупростые подалгебры особых простых алгебр Ли и выделяет среди них максимальные. Эта работа, содержащая, помимо сказанного, и много других полезных результатов, а также огромный объем фактической информации, полученной путем (выполненных вручную) вычислений, стала настольной для многих математиков. Опишем ее основные идеи и результаты.

Подалгебра полупростой алгебры Ли $\mathfrak{g}$ называется регулярной, если она нормализуется картановской подалгеброй. В частности, всякая подалгебра содержащая картановскую подалгебру (например, всякая параболическая подалгебра), является 
регулярной. $\mathrm{C}$ каждым подмножеством $\Pi_{1} \subset \Pi$ системы простых корней алгебры $\mathfrak{g}$ связана полупростая регулярная подалгебра, для которой П 1 является системой простых корней. Такие подалгебры иногда называют полупростыми подалгебрами Леви, так как они являются максимальными полупростыми подалгебрами соответствующих параболических подалгебр.

В [8] Е. Б. Дынкин предложил простой и элегантный метод нахождения всех полупростых регулярных подалгебр максимального ранга простой алгебры Ли $\mathfrak{g}^{3}$. Он основан на рассмотрении так называемой расширенной системы простых корней $\widetilde{\Pi}$, получаемой добавлением к системе простых корней П алгебры $\mathfrak{g}$ младшего корня. Система $\widetilde{\Pi}$ также удовлетворяет условию (П), но уже не является линейно независимой, а подчиняется некоторому линейному соотношению с положительными целыми коэффициентами (которые играют важную роль в теории полупростых алгебр Ли, например в описании их внутренних автоморфизмов конечного порядка). Точно так же, как система простых корней описывается схемой Дынкина, расширенная система простых корней описывается расширенной схемой Дынкина.

Выкидывая из расширенной схемы Дынкина любую вершину, мы получаем схему Дынкина некоторой полупростой (но, вообще говоря, не простой) регулярной подалгебры максимального ранга алгебры $\mathfrak{g}$, система простых корней которой получается выкидыванием одного корня из системы Пٓ. Может получиться, что эта подалгебра совпадает с алгеброй $\mathfrak{g}$ (например, если мы выкинем добавленный корень); такие случаи не следует рассматривать. Далее, расширяя аналогичным образом одну из связных компонент полученной схемы Дынкина и снова выкидывая одну из вершин расширенной схемы, мы получаем схему Дынкина некоторой меньшей полупростой регулярной подалгебры максимального ранга. Результат Дынкина состоит в том, что, продолжая этот процесс, мы в конце концов получим все полупростые регулярные подалгебры максимального ранга. Ясно, что при этом максимальные подалгебры могут получиться только на первом этапе этой процедуры, и можно доказать, что они получаются в точности тогда, когда мы выкидываем корень, коэффициент при котором в вышеупомянутом линейном соотношении является простым числом ${ }^{4}$.

Всякая полупростая регулярная подалгебра полупростой алгебры Ли $\mathfrak{g}$ является полупростой регулярной подалгеброй максимального ранга в некоторой полупростой подалгебре Леви. Таким образом можно получить список, включающий все полупростые регулярные подалгебры данной алгебры. Однако нужно еще выяснить, какие из них сопряжены друг другу при помощи внутренних автоморфизмов. Это было сделано в [8] с помощью вычислений. За пять лет до своей кончины Е. Б. Дынкин, вернувшись к группам Ли, вместе со своим научным "внуком" А. Н. Минченко выполнил работу [57], в которой они предложили красивый общий метод решения этого вопроса, основанный на введенных ими "увеличенных" ("enhanced") схемах Дынкина. Что еще более важно, с помощью этих схем они также дали общий способ определения отношений включения (с точностью до сопряженности) между полупростыми регулярными подалгебрами.

Разобравшись с регулярными подалгебрами, Е. Б. Дынкин переходит к произвольным подалгебрам. Подалгебру полупростой алгебры Ли g он называет $R$-подалгеброй, если она содержится в некоторой собственной регулярной подалгебре, и $S$-подалгеброй в противном случае. В некотором смысле $S$-подалгебры являются аналогом неприводимых линейных алгебр Ли. Из характеризации редуктивных регулярных подалгебр

\footnotetext{
${ }^{3}$ В завуалированном виде этот метод содержался в работе А. Бореля и Ж. Зибенталя [61].

${ }^{4} \mathrm{~B}[8]$ ошибочно утверждалось, что все полученные на первом этапе подалгебры являются максимальными.
} 
как подалгебр неподвижных точек диагонализуемых подгрупп присоединенной группы (группы внутренних автоморфизмов) алгебры $\mathfrak{g}$ [61] следует, что $S$-подалгебры это полупростые подалгебры, централизатор которых в группе внутренних автоморфизмов алгебры $\mathfrak{g}$ тривиален.

Ясно, что всякая полупростая подалгебра является $S$-подалгеброй в некоторой полупростой регулярной подалгебре. Тем самым, описание всех полупростых подалгебр полупростых алгебр Ли сводится (по модулю выявления сопряженных подалгебр) к описанию всех полупростых $S$-подалгебр простых алгебр Ли.

Значительную часть полупростых подалгебр особых простых алгебр Ли составляют трехмерные простые подалгебры (“трехчленные подалгебры” в терминологии Дынкина или "slㅏㄱ-тройки" в более поздней терминологии). Е.Б. Дынкин показал, что такая подалгебра с точностью до сопряженности однозначно определяется своим полупростым элементом (нормированным определенным образом), который он назвал ее характеристикой. Можно считать, что характеристика принадлежит фиксированной картановской подалгебре и является в ней доминантным элементом, т. е. значения всех простых корней на ней неотрицательны. Е. Б. Дынкин установил априори фундаментальное свойство характеристики, состоящее в том, что (при вышеуказанном соглашении) значения всех простых корней на ней равны 0,1 или 2 . После этого он нашел сначала все трехмерные простые $S$-подалгебры простых алгебр Ли (их список невелик), а затем, пользуясь классификацией полупростых регулярных подалгебр, - все трехмерные простые подалгебры особых простых алгебр Ли. Кроме того, он вычислил размерности неприводимых компонент ограничения на эти трехмерные подалгебры присоединенного представления объемлющей алгебры Ли.

С учетом теоремы Морозова-Джекобсона [86], [75] о включении любого нильпотентного элемента полупростой алгебры Ли в трехмерную простую подалгебру ${ }^{5}$ и теоремы Костанта [78] о том, что эта трехмерная подалгебра определена однозначно с точностью до сопряженности при помощи централизатора данного нильпотентного элемента ${ }^{6}$, результаты Дынкина можно интерпретировать как классификацию нильпотентных элементов особых простых алгебр Ли, хотя сам Дынкин такой вопрос не ставил. Метод Дынкина был впоследствии успешно применен для классификации нильпотентных элементов в более общих линейных представлениях простых алгебр Ли [98], [97].

Наиболее сложная и трудоемкая часть работы [8] - это классификация полупростых $S$-подалгебр ранга $>1$ в особых простых алгебрах Ли. Здесь используются все предыдущие результаты работы. Одновременно находятся все отношения включения между полупростыми $S$-подалгебрами. Далее по описанной выше схеме отсюда получается классификация всех полупростых и, наконец, всех полупростых максимальных подалгебр особых простых алгебр Ли.

Следует, однако, сказать, что если полупростые регулярные, максимальные и $S$-подалгебры были классифицированы Е. Б. Дынкиным с точностью до сопряженности, то прочие полупростые подалгебры были им классифицированы лишь с точностью до линейной сопряженности. (Две подалгебры алгебры Ли g называются линейно сопряженными, если их образы при любом линейном представлении алгебры g сопряжены в соответствующей полной линейной алгебре Ли.) Этот пробел был ликвидирован в недавней работе А.Н. Минченко [85]. Оказалось, что класс линейной сопряженности, как правило, совпадает с классом сопряженности; все исключения были в этой работе перечислены.

\footnotetext{
${ }^{5}$ Об истории доказательства этой теоремы см. [89].

${ }^{6}$ Последний факт был также доказан в неопубликованной диссертации В. В. Морозова [87]; доказательство Морозова приведено в [89].
} 
Результаты работы [8] послужили образцом для похожих результатов о подгруппах простых алгебраических групп над алгебраически замкнутыми полями положительной характеристики и простых конечных групп лиевского типа, хотя доказательства последних результатов потребовали иных методов.

Одним из важных инструментов, использованных Е. Б. Дынкиным в [8], было понятие индекса гомоморфизма $\varphi: \mathfrak{h} \rightarrow \mathfrak{g}$ простых алгебр Ли. При условии, что инвариантные скалярные умножения в алгебрах $\mathfrak{g}$ и $\mathfrak{h}$ нормированы так, что скалярный квадрат старшего корня (или, в более инвариантных терминах, соответствующего дуального корня) равен 2 , индекс $j_{\varphi}$ гомоморфизма $\varphi$ определяется из формулы

$$
(\varphi(x), \varphi(y))=j_{\varphi}(x, y) \quad \text { для любых } x, y \in \mathfrak{h} .
$$

Индекс является целым положительным числом и мультипликативен относительно композиции гомоморфизмов. Для индекса неприводимого линейного представления $\varphi: \mathfrak{g} \rightarrow \mathfrak{s l}_{n}(\mathbb{C})$ со старшим весом $\Lambda$ Е. Б. Дынкиным была получена следующая формула:

$$
j_{\varphi}=\frac{n}{\operatorname{dim} \mathfrak{g}}(\Lambda, \Lambda+g),
$$

где $g$ - сумма всех положительных корней алгебры $\mathfrak{g}$.

В работе [8] индексы применялись, во-первых, для различения изоморфных, но не сопряженных подалгебр и, во-вторых, для доказательства невозможности тех или иных вложений простых алгебр Ли. В последующем понятие индекса оказалось полезным для исследования стабилизаторов общего положения в линейных представлениях полупростых групп Ли [58], [67].

1.3. Топология компактных групп Ли. Серия работ Е. Б. Дынкина посвящена топологии компактных групп Ли. $\mathrm{K}$ тому времени была известна теорема Хопфа (которую связывают также с именами Х. Самельсона и других математиков) о том, что алгебра $H^{*}(G, \mathbb{Q})$ рациональных когомологий связной компактной группы Ли $G$ является алгеброй Грассмана с числом образующих, равным рангу группы $G$, и что в качестве системы свободных образующих этой алгебры может быть взят любой однородный базис градуированного пространства $P^{*}(G, \mathbb{Q})$ примитивных классов когомологий. Для классических компактных групп Ли свободные образующие этой алгебры были найдены Л. С. Понтрягиным. Е. Б. Дынкин [10] доказал аналогичную теорему для кольца $H^{*}(G)$ слабых целочисленных когомологий (фактора кольца целочисленных когомологий по идеалу кручения), усилив тем самым результат Хопфа. Он предложил общий метод построения базиса градуированной группы $P^{*}(G)$ примитивных целочисленных классов слабых когомологий и показал, что для классических групп этот метод приводит к классам когомологий, найденным Понтрягиным.

Каждый гомоморфизм $\Phi: H \rightarrow G$ связных компактных групп Ли индуцирует гомоморфизм градуированных групп $\Phi^{*}: P^{*}(G) \rightarrow P^{*}(H)$. Знание этого гомоморфизма важно для вычисления когомологий однородного пространства $G / \Phi(H)$. Используя связь примитивных классов когомологий с полиномиальными инвариантами присоединенного представления, Е. Б. Дынкин [11] предложил способ вычисления гомоморфизма $\Phi^{*}$ (впоследствии усовершенствованный И. З. Розенкнопом [91]). Кроме того, он доказал, что неприводимое представление $\Phi$ связной полупростой компактной группы Ли $H$ в классическую компактную линейную группу $G$ определяется гомоморфизмом $\Phi^{*}$ с точностью до умножения на автоморфизм группы $G$.

Заметим, что если $G$ и $H$ - простые группы Ли, то группы $P^{3}(G)=H^{3}(G)$ и $P^{3}(H)=H^{3}(H)$ циклические и гомоморфизм $\Phi^{3}: P^{3}(G) \rightarrow P^{3}(H)$ определяется одним целым числом, которое (с точностью до знака) совпадает с индексом гомоморфизма $d \Phi: \mathfrak{h} \rightarrow \mathfrak{g}$ соответствующих алгебр Ли (см. определение индекса выше). 
1.4. Другие работы. В одной из первых своих работ [3] Е. Б. Дынкин открыл замечательно простой способ вычисления коэффициентов в формуле Кемпбелла-Хаусдорфа. Напомним, что эта формула представляет собой выражение формального ряда $\log \left(e^{x} e^{y}\right)$ от некоммутирующих переменных $x$ и $y$ через кратные коммутаторы этих переменных и является одним из способов доказательства того фундаментального факта теории групп Ли, что локальная группа Ли однозначно определяется своей касательной алгеброй Ли. Коэффициенты этой формулы могут вычисляться рекуррентным образом, но их явный вид в то время не был известен. Е.Б. Дынкин обнаружил, что они могут быть очень просто найдены при помощи следующего доказанного им общего алгебраического утверждения.

Пусть имеется однородный многочлен степени $m$ от некоммутирующих переменных $x_{1}, \ldots, x_{n}$, про который известно, что он может быть представлен в виде линейной комбинации кратных коммутаторов этих переменных. Тогда такое представление может быть получено, если просто заменить каждое произведение $x_{i_{1}} x_{i_{2}} \cdots x_{i_{m}}$ на $m^{-1}\left[\left[\ldots\left[x_{i_{1}}, x_{i_{2}}\right], \ldots\right], x_{i_{m}}\right]$ !

Это позволило Е. Б. Дынкину обобщить теорию Ли на локальные банаховы группы и алгебры Ли над произвольными полными нормированными полями нулевой характеристики [4], что составило его кандидатскую диссертацию.

В 1960-е годы Е. Б. Дынкин выполнил несколько работ по броуновскому движению в симметрических пространствах, соединивших две темы его исследований - группы Ли и теорию вероятностей. Об этих работах будет рассказано в следующем разделе.

\section{2. Работы Е. Б. Дынкина по теории вероятностей}

2.1. Ранние работы по теории вероятностей. Интерес Е. Б. Дынкина к теории вероятностей возник еще в студенческие годы. Его первая работа [1], совместно с Н. А. Дмитриевым, содержала решение задачи о характеристических корнях стохастических матриц, поставленной Колмогоровым на своем семинаре. Оба автора были в это время студентами пятого курса мехмата МГУ. Защитив кандидатскую диссертацию, Е. Б. Дынкин поступил на работу на кафедру теории вероятностей мехмата, руководимую в те годы А.Н. Колмогоровым. Он читает лекции как по теории вероятностей, так и по алгебре и анализу, и участвует в работе семинаров по теории вероятностей и математической статистике. Из работ этого периода отметим статью [6], посвященную достаточным статистикам. Здесь устанавливаются критерии существования необходимых и достаточных статистик в зависимости от размерности некоторого вспомогательного линейного пространства; если эта размерность превышает объем выборки, нетривиальных достаточных статистик не существует. Почти тридцать лет спустя Е. Б. Дынкин вернулся к этой теме в своей работе [36], связав эти результаты с более поздними результатами по граничному поведению марковских процессов.

Упомянем также работу [12], в которой изучается поведение сумм независимых случайных величин в случае, когда слагаемые неотрицательны и имеют бесконечное математическое ожидание.

2.2. Марковские процессы: основания теории. О роли Е. Б. Дынкина в становлении теории марковских процессов написано несколько статей, в частности статья П.-А. Мейера [84] и две прекрасные статьи А. А. Юшкевича - [100] и в особенности [101]. Понятно, что здесь нам придется повторить многое из упомянутого в этих статьях.

До середины 1950-х годов современного понятия марковского процесса не существовало. Отдельные классы марковских процессов изучались главным образом с помощью аналитических методов, либо в связи с дифференциальными уравнениями 
второго порядка (Колмогоров), либо с помощью полугрупп операторов (Феллер). Исследование свойств траекторий процесса в некотором смысле началось в конце 1940-х годов с процессов со счетным пространством состояний и поначалу не вызвало воодушевления. Как отмечает Юшкевич [100], еще в 1951 г. Колмогоров привел другое, чисто аналитическое доказательство некоторых результатов Дуба, упомянув, что это доказательство проще, чем доказательство Дуба, использующее меры в функциональных пространствах.

Как указывает Юшкевич, интерес Е. Б. Дынкина к марковским процессам возник в ходе чтения соответствующего спецкурса. В работе [9], первой из его работ в этом направлении, дается критерий существования марковского процесса с непрерывными траекториями в терминах его переходной функции $p(s, x ; t, \Gamma)$. А именно, если для любого $\varepsilon>0$ предел

$$
\lim _{\delta \rightarrow 0} \sup _{t: s<t<s+\delta} p\left(s, x ; t, \overline{U_{\varepsilon}(x)}\right)
$$

равен нулю, причем сходимость равномерна по $x$, то существует версия этого процесса, с вероятностью единица являющаяся непрерывной справа без разрывов второго рода (здесь $\overline{U_{\varepsilon}(x)}$ - дополнение $\varepsilon$-окрестности точки $x$ ). Если же выражение под знаком предела стремится к нулю как $o(\delta)$, то процесс можно сделать непрерывным. Близкий результат был год спустя независимо получен Дж. Кинни [77] (у Кинни критерий непрерывности совпадал с критерием, предложенным в [9], а критерий отсутствия разрывов второго рода был несколько слабее). Тем не менее соответствующие результаты вошли в теорию как критерий Дынкина-Кинни.

К 1954 г. Е. Б. Дынкин практически полностью сосредотачивается на теории марковских процессов. Организованный им семинар по теории марковских процессов привлекает аспирантов и студентов, постепенно Е. Б. Дынкин становится одним из основателей современной теории марковских процессов и одним из лидеров московской вероятностной школы. Он выпускает две монографии [14], [17], посвященные теории марковских процессов, в которых приводятся как его собственые результаты, так и результаты других участников его семинара, в том числе А. Д. Вентцеля, В.А. Волконского, И. В. Гирсанова, Л.В. Серегина, М.И. Фрейдлина, Р. З. Хасьминского, М. Г. Шура, А. А. Юшкевича. Здесь мы сосредоточимся на предложенных Е. Б. Дынкиным ключевых идеях, понятиях и конструкциях, оказавших большое влияние на развитие теории в целом (см. [14], [17]).

2.2.1. Определение марковского процесса. Зарождение теории марковских процессов связано с именами А. А. Маркова, Л. Башелье, Н. Винера, А. Н. Колмогорова, А.Я. Хинчина. Долгое время в центре внимания теории была переходная функция и связанные с ней операторы в пространстве функций. Систематическое исследование свойств траекторий процесса началось с работ Дж. Л. Дуба [65]. При этом под марковским процессом понимался случайный процесс, обладающий марковским свойством - условной независимостью прошлого и будущего при известном настоящем (так называемый марковский процесс в смысле Дуба). Однако для развитой теории требовалось одновременно рассматривать процессы, отвечающие различным начальным состояниям и начальным моментам. Эти процессы должны были быть согласованы друг с другом, в частности для того, чтобы было возможно работать с условными распределениями при известном настоящем. В ряде примеров естественным образом появлялись также процессы со случайным моментом обрыва. С учетом этого Е. Б. Дынкин определяет (неоднородный) марковский процесс как семейство вероятностных мер $P_{s, x}$ на одном и том же пространстве элементарных событий $(\Omega, \mathscr{F})$. Определение включает в себя момент обрыва $\zeta$ и $\sigma$-алгебры $\mathscr{F}_{t}^{s}$, которые интерпретируются как совокупности событий, наблюдаемых на интервале времени $[s, t]$, и по 
отношению к которым выполняется марковское свойство ${ }^{7}$. Однородные (по времени) марковские процессы рассматриваются как важный частный случай, в их определение входит также оператор сдвига $\theta_{t}$.

2.2.2. Характеристический оператор. Для описания локального поведения марковского процесса обычно использовался инфинитезимальный оператор полугруппы операторов, связанных с переходной функцией, т. е. предел

$$
A f(x)=\lim _{t \rightarrow 0} t^{-1}\left(P_{x} f\left(\xi_{t}\right)-f(x)\right) .
$$

Однако этот оператор характеризует локальное поведение процесса во времени, а не в пространстве. Е. Б. Дынкин предложил взамен характеристический оператор

$$
\mathfrak{A} f(x)=\lim _{\varepsilon \rightarrow 0} \frac{P_{x} f\left(\xi_{\tau_{\varepsilon}}\right)-f(x)}{P_{x} \tau_{\varepsilon}},
$$

определяемый через момент первого выхода $\tau_{\varepsilon}$ из $\varepsilon$-окрестности точки $x$, фактически заменив предельный переход по времени предельным переходом по пространству (здесь и далее $P_{x}$ - математическое ожидание, вычисленное в предположении, что процесс начинается в точке $x$ ). Характеристический оператор оказался намного более естественным объектом - например, он позволил дать вероятностный эквивалент разработанной незадолго до этого В. Феллером (аналитической) характеризации одномерных диффузий.

2.2.3. Строго марковское свойство. В простейшей форме строго марковское свойство означает, что для определенного класса случайных моментов $\tau$ случайный процесс $\tilde{\xi}_{t}=\xi_{\tau+t}$ также является марковским процессом с той же самой переходной функцией, что и исходный процесс. При этом класс допустимых моментов $\tau$, так называемых марковских моментов (они же моменты остановки), включает в себя моменты первого достижения $\tau_{\Gamma}=\inf \left\{t: \xi_{t} \in \Gamma\right\}$, где $\Gamma-$ подмножество пространства состояний. Для процессов с дискретным временем строго марковское свойство легко выводится из обычного марковского свойства. В случае непрерывного времени ситуация много сложнее.

Строго марковское свойство неоднократно использовалось в теории без каких-либо обоснований. Классическим примером может служить так называемый принцип отражения при изучении свойств максимума броуновского движения. Первое аккуратно доказанное утверждение в этом направлении содержится в работе Дуба [65], где строго марковское свойство доказано для определенного класса марковских цепей с непрерывным временем. В [66] Дуб распространяет строго марковское свойство на $n$-мерное броуновское движение. При этом и в [65], и в [66] он отмечает, что это частный случай гораздо более общего утверждения, которое, однако, так и не было сформулировано и опубликовано.

В современной форме строго марковское свойство устанавлено в работе Е. Б. Дынкина и А. А. Юшкевича [13] для непрерывных справа феллеровских процессов ${ }^{8}$ в произвольном метрическом пространстве. В частности, в [13] вводятся такие объекты, как $\sigma$-алгебра событий $\mathscr{F}_{\tau}$, описывающая “прошлое” процесса до момента $\tau$. Одновременно, но для более узких классов процессов или же в более ограничительной форме, подобные результаты были получены Дж. А. Хантом [72], Д. Рэем [90], К.Л. Чжуном [63] и Р. Блюменталем [60].

Семнадцать лет спустя Е. Б. Дынкин возвращается к этой теме в [33].

${ }^{7} \sigma$-алгебры $\mathscr{F}_{t}^{s}$ содержат $\sigma$-алгебры, порожденные траекториями процесса на интервале $[s, t]$, но не обязательно совпадают с ними.

${ }^{8}$ Марковский процесс $\xi_{t}$ называется феллеровским, если операторы $P_{t} f(x)=P_{x} f\left(\xi_{t}\right)$ переводят непрерывные функции в непрерывные. 
2.2.4. Стандартные процессы. Понятно, что класс марковских процессов очень широк и включает в себя множество уродливых примеров. Естественно задать вопрос, какие же свойства марковского процесса позволяют исключить нежелательные примеры, не исключая при этом ничего существенного. В качестве ответа Е. Б. Дынкин предложил понятие стандартного процесса. Это (однородный) непрерывный справа без разрывов второго рода строго марковский процесс, принимающий значения в локально компактном хаусдорфовом пространстве со счетной базой. Кроме того, предполагается, что $\sigma$-алгебры $\mathscr{F}_{t}=\mathscr{F}_{t}^{0}$ непрерывны справа по $t$ в том смысле что $\mathscr{F}_{s}=\bigcap_{t>s} \mathscr{F}_{t}$. И наконец, если $\left(\tau_{n}\right), \tau_{n}<\tau, \tau_{n} \uparrow \tau,-$ монотонно возрастающая последовательность марковских моментов, то $\xi_{\tau_{n}} \rightarrow \xi_{\tau}$ с вероятностью единица (так называемая квазинепрерывность слева). В западной литературе похожее место занимали процессы Ханта, для которых квазинепрерывность слева верна также и в момент обрыва процесса (значение стандартного процесса в момент обрыва не определено и соответствующее свойство не имеет смысла, а процесс Ханта определен и в момент обрыва). Много позднее, в середине 1970-х годов, появились процессы Рэя, для которых свойство квазинепрерывности слева было заменено так называемым умеренно марковским свойством.

2.2.5. Естественная топология и эксцессивные функции. Понятие тонкой топологии восходит к работе А. Картана [76], определившего ее как слабейшую топологию, в которой непрерывны все субгармонические функции. Позднее Дж. Л. Дуб [66] показал, что если точка $x$ на плоскости не принадлежит замыканию множества $A$ в топологии Картана, то момент $\tau_{A}$ достижения этого множества броуновским движением, начинающимся из точки $x$, с вероятностью единица строго положителен. Для стандартных марковских процессов множество $A$ называется тонко открытым, если марковский процесс, начинающийся из любой точки множества $A$, с вероятностью единица не достигает дополнения множества $A$ за бесконечно малое время. В [17] строится база тонкой топологии и доказывается, что функция непрерывна в тонкой топологии тогда и только тогда, когда она с вероятностью единица непрерывна справа вдоль траекторий процесса (последний результат принадлежит И. В. Гирсанову и М.Г. Шуру).

2.2.6. Аддитивные функционалы. Аддитивный функционал - это семейство случайных величин $A_{s t}, s<t$, измеримых относительно $\sigma$-алгебр $\mathscr{F}_{t}^{s}$ и таких, что $A_{s t}+A_{t u}=A_{s u}$, если $s<t<u$. Простейшим примером может служить функционал интегрального типа

$$
A_{s t}=\int_{s}^{t} f\left(u, \xi_{u}\right) d u
$$

где $f$ - некоторая функция. Функционалы интегрального типа изучались и ранее, общее определение было предложено Е. Б. Дынкиным в [15]. Неотрицательный аддитивный функционал можно интерпретировать как временную шкалу, зависящую от траектории процесса. В частности, сюда попадает локальное время на подмножестве пространства состояний и многие другие примеры. Аддитивные функционалы и по сей день являются важным инструментом в теории марковских процессов. Так, например, они в полной мере необходимы при изучении свойств суперпроцессов (см. [45], [47], [48]).

2.2.7. Преобразования марковских процессов. Преобразования, позволяющие, начиная с марковского процесса, построить новый марковский процесс с желаемыми свойствами, изучались многими авторами. В число таких преобразований входят случайная замена времени, замена меры, убивание, $h$-преобразование и многое другое. Предложенные Е.Б. Дынкиным $(\alpha, \xi)$-подпроцессы объединили вместе и убивание, и замену меры. Грубо говоря, мультипликативный функционал $\alpha$ ответственен за 
убивание, а функция $\xi$ отвечает за замену меры (точнее, это то, что происходит, если присутствует только одна из компонент).

2.2.8. Непрерывные одномерные строго марковские процессы. Структура одномерных непрерывных процессов привлекла к себе внимание благодаря работе В. Феллера [69], в которой изучаются инфинитезимальные операторы непрерывных строго марковских процессов и доказывается что эти операторы в определенном смысле являются псевдодифференциальными операторами второго порядка. Однако работы Феллера были чисто аналитическими. В [17] аналогичные результаты устанавливаются чисто вероятностными методами, с использованием характеристических операторов вместо инфинитезимальных.

2.3. Границы Мартина и продолжения марковского процесса. В начале 1960-х годов Е.Б. Дынкин обращает свое внимание на границы Мартина. На важность границ Мартина для теории марковских процессов первым указал еще Дж.Л. Дуб в конце 1940-х годов. Позднее Дж. А. Хант показал, что связанные с границей Мартина аналитические результаты могут быть получены чисто вероятностными методами. Е. Б. Дынкин впервые обращает свое внимание на связанные с границами Мартина проблемы в начале 1960-х годов. В приглашенном докладе на Международном математическом конгрессе в Стокгольме $[18]^{9}$, в частности, содержится описание предельного поведения броуновского движения в пространстве эллипсоидов, а именно вычисляется соответствующая граница Мартина (подробные доказательства были опубликованы позднее в [21]). Здесь же он сформулировал гипотезу о связи между границей Мартина для риманова многообразия и его кривизной. Как отмечает А. А. Юшкевич [100], эта гипотеза была подтверждена в 1980-х годах трудами М. Т. Андерсона, Д. Сулливана, Р. Шоэна и других авторов. Сюда примыкает и совместная с М. Б. Малютовым работа [16], в которой изучаются свойства границы Мартина для случайного блуждания на неабелевой группе с конечным числом образующих. Как указано в [100], эти результаты использовались далее Г. Фюрстенбергом и другими авторами.

Несколько работ Е. Б. Дынкина посвящены так называемой краевой задаче с наклонной производной для уравнения Лапласа (эти исследования подытожены в [20]). Аналитически задача формулируется следующим образом. Пусть $D$ - гладкая ограниченная область на плоскости, и пусть $v$ - векторное поле, заданное на границе области $\partial D$. Требуется найти все гармонические функции $h$ в области $D$, удовлетворяющие на границе области условию $\partial h / \partial v=0$. Эта задача нетривиальна, если поле $v$ может быть касательным к границе в некоторых точках. С точки зрения теории марковских процессов речь идет о поведении броуновского движения в ограниченной гладкой области с отражением на границе в направлении поля $v$. Эта задача изучалась многими авторами, начиная с Пуанкаре. А.-М. Льенар предложил простой способ описания всех решений этой задачи, гладких вплоть до границы. Е. Б. Дынкину, в предположении, что поле является касательным к границе в конечном числе точек, удалось описать все неотрицательные решения этой задачи, причем в точках, где поле становится касательным, допускаются любые особенности.

2.3.1. Граничные условия для марковских процессов. К этой тематике примыкает задача описания всех марковских процессов с заданными локальными характеристиками (в общем случае, с одним и тем же характеристическим оператором). В какой-то форме этим интересовался еще Дж. Л. Дуб в 1940-х годах. Поскольку характеристический оператор описывает поведение процесса вплоть до ухода на бесконечность, или же до выхода на границу области, или же - в общем случае - до выхода на границу Мартина, речь идет о дополнительных граничных условиях на

\footnotetext{
9 Доклад Дынкина на конгрессе был представлен А. Н. Колмогоровым.
} 
границе Мартина, которые позволяют однозначно охарактеризовать процесс. В частности, А. Д. Вентцель [94] исследовал общие граничные условия для многомерных диффузионных процессов в гладкой области. В. Феллер [70] рассматривал аналогичную задачу для марковских процессов с дискретным пространством состояний, но ему удалось лишь описать процессы, удовлетворяющие дополнительному условию. Е. Б. Дынкин в [23] смог дать полный ответ на этот вопрос, без каких-либо дополнительных ограничений. В [22] он рассматривает аналогичную задачу для диффузионных процессов с наклонной производной.

2.3.2. Странствия марковского процесса. Пусть $X_{t}-$ стандартный марковский процесс в пространстве $E$, и пусть $D$ - открытое подмножество $E$. Множество моментов времени, для которых $X_{t} \in D$, распадается на открытые справа интервалы. Соответствующие куски траектории называются странствиями. Понятие странствия обобщает рассматривавшееся ранее К. Ито понятие экскурсии. В работе [31] (см. также [25] и [26]) Е. Б. Дынкин изучает странствия марковского процесса и выводит так называемую формулу странствий для математического ожидания некоторой суммы функционалов от странствий процесса. Эта формула была позднее независимо получена Р. К. Гетуром, М. Шарпом и Б. Мезоннёвом и использовалась далее под названием "last exit decomposition" - разложение, связанное с моментом последнего выхода.

2.4. Диффузия в пространстве тензоров. Развивая идеи Ито [74], Е. Б. Дынкин [24] рассмотрел диффузии в пространстве тензоров и связанное с этим понятие случайного параллельного переноса. Как отмечено в [100], в 1980-х годах этот подход был распространен Л. Шварцем, Р.-А. Мейером и И. Шигекавой на семимартингалы и нашел широкое применение в стохастической дифференциальной геометрии.

2.5. Оптимальная остановка марковских процессов. Отметим несколько работ, связанных с задачей об оптимальной остановке. Е. Б. Дынкин обращался к этой тематике не однажды. В работе [19] он привел простое и изящное решение этой задачи в терминах эксцессивной мажоранты целевой функции. Игровому варианту задачи об оптимальной остановке посвящена работа [29]. Недавно описанный там подход нашел широкое применение в математической экономике под названием игр Дынкина ("Dynkin games"). Обзоры других работ, так или иначе связанных с математической экономикой, можно найти в [35] и [68].

2.6. Граничное поведение марковских процессов. Неоднородные марковские процессы в общих пространствах состояний. В конце 1960-х годов Е. Б. Дынкин сосредотачивается на общей граничной теории для марковских процессов.

2.6.1. Разложение эксцессивных функций по крайним точкам. Как известно, неотрицательные гармонические функции в области могут быть однозначно представлены в виде интеграла от ядра Мартина (или же ядра Пуассона, если область гладкая). Это утверждение можно также интерпретировать как существование и единственность разложения неотрицательных гармонических функций по крайним точкам.

В общей теории марковских процессов роль неотрицательных супергармонических функций играют эксцессивные функции. Возникает естественный вопрос: при каких предположениях это утверждение сохраняет силу для эксцессивных функций? Ответ на этот вопрос тесно связан с такими вещами, как границы Мартина и финальное поведение марковского процесса.

Первое серьезное продвижение в этом направлении было сделано Дж. А. Хантом [73]. В этой большой работе в предположении, что исходный процесс строго марковский и непрерывен справа, строится вероятностный аналог классической теории 
потенциала (вероятностная теория превращается в классическую теорию потенциала, если исходный процесс - броуновское движение).

Е.Б. Дынкин заинтересовался этим кругом вопросов во второй половине 1960-х годов. Он публикует ряд работ на эту тему, в том числе две большие работы [27] и [28], первая из которых посвящена марковским цепям с непрерывным временем, а вторая - общим марковским процессам. Тем не менее полученные результаты не удовлетворили его - часть предположений явно носила технический характер.

После нескольких попыток избавиться от технических ограничений, Е. Б. Дынкин кардинально изменил подход [30], [32]. Во-первых, он рассматривает неоднородные марковские процессы и доказывает существование и единственность разложения неоднородных эксцессивных мер и неоднородных эксцессивных функций по крайним точкам. Для однородных процессов отсюда без больших проблем удается получить разложение однородных мер и функций по крайним однородным мерам и функциям. Во-вторых, он сначала устанавливает разложение по крайним точкам для эксцессивных мер, а затем сводит эксцессивные функции к коэксцессивным мерам - эксцессивным мерам для дуальной полугруппы. Оказывается, что при этом практически все ограничения, касающиеся свойств траекторий процесса, исчезают, не нужна даже топология в пространстве состояний. Более того, еще один маленький шаг позволил C. Е. Кузнецову [80] вывести отсюда уже и необходимые и достаточные условия для разложения эксцессивных функций по крайним.

Столь значительный выигрыш в общности был достигнут не только за счет смены стратегии, но и благодаря нескольким принципиальным идеям и техническим результатам. Во-первых, Е.Б. Дынкин связал эксцессивные меры с одномерными распределеними марковского процесса с заданной переходной функцией. Для этого потребовалось ввести новый объект - марковские процессы со случайными моментами рождения $\alpha$ и гибели $\beta$. Подобные процессы строятся по своим двумерным распределениям как меры на пространстве траекторий со случайным интервалом жизни. Такое построение было осуществлено Е.Б. Дынкиным в случае, когда в результате получается вероятностная мера, и позднее распространено С.Е. Кузнецовым на случай $\sigma$-конечных мер [79]. Далее, используя тот простой факт, что переходная функция марковского процесса вдоль траекторий процесса является неотрицательным мартингалом (с моментом рождения $\alpha$ ), Е. Б. Дынкин строит пространство входов для марковского процесса через правые пределы таких мартингалов в точке $\alpha$ и устанавливает взаимно однозначное соответствие между вероятностными мерами на пространстве входов и марковскими процессами с данной переходной функцией, т. е. с эксцессивными мерами. Позднее, в работе [36], он связал этот результат со своей давней работой о достаточных статистиках [6]. Предполагая дополнительно существование переходной плотности и, соответственно, копереходной функции (она же дуальная переходная функция), он далее сводит разложение по крайним эксцессивных функций к уже установленному разложению по крайним эксцессивных мер для дуальной переходной функции.

2.6.2. Регулярные процессы. После успеха с эксцессивными функциями естественно было попытаться отказаться от топологических предположений и в других разделах теории. Этому посвящены работы [33] и [34]. В первой из них строится общая теория марковских процессов со случайными моментами рождения и гибели. Такие свойства, как непрерывность справа траекторий процесса, заменяются непрерывностью справа переходной функции вдоль траекторий процесса (так называемые регулярные марковские процессы). Для регулярных процессов доказывается строго марковское свойство. Кроме того, эксцессивные функции также непрерывны справа вдоль траекторий процесса. Во второй работе исходной точкой служит обладающая марковским свойством система $\sigma$-алгебр $\mathscr{F}(s, t)$ событий, наблюдаемых на интервале времени $(s, t)$, и строится симметричная относительно обращения времени теория. 
Важную роль в восстановлении симметрии прошлого и будущего играет так называемая копереходная функция. Это понятие использовалось и в обсуждаемой выше задаче о разложении эксцессивных функций по крайним [30], [32]. Одновременное рассмотрение переходной и копереходной функций в некотором смысле эквивалентно рассмотрению пары дуальных марковских процессов ${ }^{10}$.

2.7. Случайные поля, формы Дирихле и самопересечения траекторий броуновского движения. В конце 1970-х годов Е. Б. Дынкин обращает внимание на новую для себя область - случайные поля. Если случайный процесс можно определить как семейство случайных величин $X_{t}$, где параметр $t$ одномерен и интерпретируется как время, то случайное поле - это семейство случайных величин $Z_{x}$, где точка $x$ интерпретируется как точка пространства (или некоторого множества $E$ ). В зависимости от ситуации, в качестве множества $E$ может выступать подмножество $\mathbb{R}^{n}$, или же пространство гладких функций на $\mathbb{R}^{n}$, или же пространство конечных мер на подмножестве $\mathbb{R}^{n}$, или что-нибудь другое.

Первой работой Е. Б. Дынкина в этом направлении была статья [37]. Исходным объектом здесь является марковский процесс с пространством состояний $E$, переходной плотностью $p(t, x, y)$ и функцией Грина $g(x, y)=\int_{0}^{\infty} p(t, x, y) d t$. В предположении, что переходная плотность симметрична, Е. Б. Дынкин строит гауссовское случайное поле с нулевым средним $\varphi_{\mu}$, для которого

$$
\mathrm{E} \varphi_{\mu} \varphi_{\nu}=\int_{E \times E} g(x, y) \mu(d x) \nu(d y) .
$$

Здесь $\mu, \nu$ - меры на $E$, удовлетворяющие определенному условию конечности. Без пространства мер можно и обойтись, если функция Грина всюду конечна, но для большинства интересных примеров это не так. Е. Б. Дынкин доказывает, что построеннное случайное поле является марковским, и вычисляет условные математические ожидания значений поля при известных значениях поля на подобласти $E$. Аналогичная конструкция возможна и для нескольких марковских процессов. Возникающий таким образом объект тесно связан с так называемыми формами Дирихле. Формы Дирихле и пространства Дирихле берут свое начало в работе А. Берлинга и Ж. Дени [59]. Первоначально эти понятия были связаны с оператором Лапласа и броуновским движением. М. Фукушима, М. Сильверстейн и другие авторы распространили соответствующую теорию на случай непрерывных справа марковских процессов с симметричной переходной функцией (см., например, [71]). При этом предполагалось, что пространство состояний наделено топологией и, более того, соответствующее пространство Дирихле содержит достаточно много непрерывных функций. В серии работ (в их числе [38], [39]) Е. Б. Дынкин развил альтернативную и в некоторых отношениях более общую теорию пространств Дирихле, связанных с регулярными марковскими процессами, имеющими симметричную переходную плотность. При этом наличие топологии не предполагается, а класс непрерывных функций заменяется на класс функций, непрерывных справа вдоль траекторий процесса.

В работе [40] Е. Б. Дынкин рассматривает марковскую цепь с непрерывным временем на конечном или счетном множестве $E$ и связывает с ней два случайных поля на $E$, а именно $\tau_{x}$ - время пребывания в состоянии $x$ и $\nu_{x}$ - число посещений состояния $x$ (накладываются условия, при которых оба поля конечны с вероятностью единица). С помощью этих полей он изучает свойства случайного поля на $E$ при условии, что гамильтониан поля определенным образом связан с матрицей интенсивностей рассматриваемой цепи.

\footnotetext{
${ }^{10}$ Понятие копереходной функции активно используется в эргодической теории и сегодня (см., например, [95] и [96]).
} 
В [41] этот подход распространяется на общие марковские процессы $X_{t}$ с симметричной переходной плотностью. В этой ситуации время пребывания $T_{x}$ в состоянии $x$ становится обобщенным случайным полем, его можно определить как интеграл $T_{B}=\int_{0}^{\zeta} 1_{B}\left(X_{t}\right) d t$, где $B$ - подмножество пространства состояний, а $\zeta$ - момент обрыва процесса. Центральное место в работе занимает тождество, связывающее гауссовское случайное поле, построенное в [37], и поле $T_{x}$. Это тождество позволяет, с одной стороны, использовать марковские процессы для изучения негауссовских случайных полей, возникающих в квантовой теории поля и подобных построенным в [92]. С другой стороны, оказывается возможным использовать технику квантовых случайных полей, например фейнмановские диаграммы, для изучения локальных времен, самопересечений и кратных точек марковского процесса.

Исследованию самопересечений траекторий броуновского движения посвящен большой цикл работ Е. Б. Дынкина, мы упомянем здесь лишь две из них [42], [43]. Интерес к самопересечениям броуновских траекторий разгорелся с новой силой после работы К. Симанзика [93], в которой он предложил в качестве модели квантового поля облако броуновских частиц, взаимодействующих между собой только в моменты пересечения траекторий. В [42] с некоторым классом мер связываются случайные меры, сосредоточенные на броуновской траектории, а также случайные меры, сосредоточенные на множестве кратных точек броуновской траектории, т. е. точек, посещаемых определенное число раз. Возникающий объект можно рассматривать как обобщенное случайное поле со значениями в множестве случайных мер. Меры на траектории броуновского движения в некотором смысле эквивалентны аддитивным функционалам, меры на множестве кратных точек существуют лишь в размерностях, не превосходящих трех. В частности, на плоскости сюда включаются так называемые двумерные броуновские петли. В [43] аналогичные объекты строятся сначала для случайного блуждания, аппроксимирующего броуновское движение, и доказывается существование соответствующих пределов.

2.8. Работы по теории суперпроцессов. Под суперпроцессами подразумеваются марковские процессы со значениями в пространстве мер, которые можно интерпретировать как вероятностные модели поведения облака ветвящихся частиц. Впервые процессы такого рода были построены Ш. Ватанабе [99] и Д. Доусоном [64]. Чтобы прояснить их интуитивный смысл, приведем простейший пример. Пусть в пространстве независимо движется некоторое количество броуновских частиц. В момент времени $\beta$, где $\beta$ - малый параметр, частицы умирают и каждая из них независимо с вероятностью $1 / 2$ оставляет после себя двух потомков (или ни одного). Вновь образованные частицы снова независимо движутся в пространстве в соответствии с законом броуновского движения и умирают в момент времени $2 \beta$, снова оставляя после себя случайное число потомков, и т. д. Как известно, подобная система частиц с вероятностью единица исчезает за конечное (случайное) число шагов.

Будем считать, что масса каждой частицы равна $\beta$. Расположение частиц в момент времени $t$ можно интерпретировать как дискретную меру $X_{t}^{\beta}$ (распределение массы в пространстве). Остается перейти к пределу, когда $\beta \rightarrow 0$. При этом, для того чтобы получить нетривиальный предельный процесс, нужно одновременно устремить к бесконечности начальное число частиц таким образом, чтобы начальное распределение массы $X_{0}^{\beta}$ стремилось к некоторой конечной мере $\mu$. В результате возникает случайный процесс $\left(X_{t}, P_{\mu}\right)$ со значениями в пространстве конечных мер (здесь $X_{t}-$ траектория процесса в пространстве мер, а $P_{\mu}$ - вероятностная мера, описывающая поведение процесса, начинающегося с начального распределения массы $\mu$ ). Получающийся процесс носит имя суперброуновского движения Доусона-Ватанабе, хотя в работе Ватанабе стартовой точкой служило не броуновское движение, а дискретная цепь Маркова, и в обоих работах [99] и [64] процесс строился аналитически, а вовсе не описанным выше предельным переходом. 
Как известно, броуновское движение связано с оператором Лапласа (оператор $\frac{1}{2} \Delta$ является его инфинитезимальным оператором). В частности, если $D \subset \mathbb{R}^{n}$ - ограниченная гладкая область, $\varphi(x)$ - непрерывная функция на границе $\partial D$ области $D$, а $\xi_{t}-$ броуновское движение в области $D$, остановленное в случайный момент $\tau$ выхода из области, то функция $h(x)=P_{x} h\left(\xi_{\tau}\right)$ является гармонической функцией, равной $\varphi$ на границе области $\partial D$. Другими словами, функция $h(x)$ является решением краевой задачи $\Delta h=0$ в области $D$ с условием $h=\varphi$ на границе.

В свою очередь, суперброуновское движение связано с нелинейным дифференциальным уравнением $\Delta u=u^{2}$. Для того чтобы сформулировать соответствующее утверждение, вернемся к описанной выше ветвящейся системе частиц и предположим, что попадающие на границу области $D$ частицы мгновенно замораживаются и больше не участвуют в эволюции системы. Обозначим $X_{D}^{\beta}$ распределение массы на границе области. При предельном переходе $\beta \rightarrow 0$ мера $X_{D}^{\beta}$ сходится к пределу конечной случайной мере $X_{D}$, которую можно интерпретировать как массу, осевшую на границе области $D$. Пусть, как и выше, $\varphi(x)$ - непрерывная функция на границе $\partial D$ области $D$. Обозначим $\left\langle h, X_{D}\right\rangle$ интеграл функции $h$ по мере $X_{D}$. Рассмотрим функцию

$$
u(x)=-\log P_{\delta_{x}} e^{-\left\langle h, X_{D}\right\rangle}
$$

где $P_{\delta_{x}}$ - математическое ожидание, вычисленное в предположении, что процесс начинается с единичной массы, сосредоточенной в точке $x$. Оказывается, что функция $u(x)$ является решением краевой задачи $\Delta u=u^{2}$ в области $D$ с условием $u=\varphi$ на границе.

2.8.1. Построение суперпроцессов. Большая группа работ Е. Б. Дынкина по суперпроцессам посвящена построению суперпроцесса и исследованию его свойств. Действительно, в описанной выше конструкции броуновское движение можно заменить на произвольный марковский случайный процесс, время жизни частиц можно сделать случайным, контролируемым подходящим аддитивным функционалом, закон распределения числа потомков может зависеть от положения в пространстве, а также от времени, и впридачу потомки могут быть как-то распределены в пространстве. Возникает вопрос, при каких условиях можно получить нетривиальный предельный процесс, а также какими свойствами обладают подобные процессы и можно ли независимо описать класс таких процессов через их свойства. Ответам на эти и другие вопросы посвящена монография [44]. В ней доказывается, что построение суперпроцесса возможно, если исходный процесс является непрерывным справа строго марковским процессом без разрывов второго рода, а упомянутый выше аддитивный функционал и характеристики закона ветвления удовлетворяют определенным условиям конечности ([44; гл. 5]; к сожалению, этот результат слишком громоздок, чтобы привести его здесь). Возникающий класс процессов можно охарактеризовать как класс мерозначных марковских процессов, обладающих следующим свойством: если начальную меру $\mu$ представить как сумму двух мер $\mu_{1}+\mu_{2}$, то распределение процесса, соответствующего начальному состоянию $\mu$, совпадает с распределением суммы двух независимых процессов, соответствующих начальным состояниям $\mu_{1}$ и $\mu_{2}[45]$.

Рассмотрим простейшую ситуацию, когда исходный процесс является диффузией, ветвление локально и характеристики ветвления не зависят от положения в пространстве, а математическое ожидание числа потомков не превосходит единицы; тогда возникающий процесс связан с нелинейным дифференциальным уравнением

$$
L u=\psi(u),
$$

где эллиптический оператор второго порядка $L$ описывает диффузию, а функция $\psi(u)$ характеризует ветвление. Возникающий при этом класс функций $\psi(u)$ дается формулой

$$
\psi(u)=b u^{2}+\int_{0}^{\infty}\left(e^{-\lambda u}-1+\lambda u\right) n(d \lambda)
$$


в которой легко узнать формулу Леви-Хинчина из теории безгранично делимых распределений. В частности, класс функций $\psi(u)$ включает в себя функции $\psi(u)=u^{\alpha}$, $1<\alpha \leqslant 2$.

2.8.2. Суперпроцессы, полярные множества и положительные решения уравнения $\Delta u=u^{\alpha}$ в области. Большой цикл работ Е. Б. Дынкина посвящен изучению свойств положительных решений уравнения $\Delta u=u^{\alpha}, 1<\alpha \leqslant 2$, в области, с помощью соответствующего суперброуновского движения ${ }^{11}$.

Мы начнем с характеризации класса полярных множеств. Множество Г называется полярным для марковского процесса $\xi_{t}$, если процесс $\xi_{t}$ с вероятностью единица никогда не посещает множество Г. Применительно к суперпроцессу $X_{t}$ это определение приходится модифицировать следующим образом. Рассмотрим носитель (range) суперпроцесса - наименьшее замкнутое множество $R$, содержащее все носители мер $X_{t}$. Множество $\Gamma$ называется полярным для суперпроцесса $X_{t}$, если носитель $R$ суперпроцесса с вероятностью единица не пересекается с множеством $\Gamma$.

Класс полярных множеств для броуновского движения можно охарактеризовать также как класс устранимых особенностей для уравнения Лапласа. А именно, множество $Г$ - устранимая особенность, если любая ограниченная функция, гармоническая в области $U \backslash \Gamma$, где $U$ - открытая окрестность $\Gamma$, может быть продолжена по непрерывности до функции, гармонической во всей области $U$. Полярные множества можно также описать как множества нулевой емкости.

Интерес Е. Б. Дынкина к этому вопросу возник в связи с работой Ж.-Ф. Ле Галля [81], в которой приводилась характеризация устранимых особенностей для уравнения $\Delta u=u^{2}$ в области, одновременно как полярных множеств и как множеств определенной емкости нуль ${ }^{12}$. Е. Б. Дынкину совместно с С. Е. Кузнецовым [46] удалось получить аналогичные результаты для суперброуновского движения, соответствующего случаю $\psi(u)=u^{\alpha}, 1<\alpha \leqslant 2$. А именно, класс полярных множеств можно охарактеризовать как класс множеств определенной емкости нуль (это так называемая бесселевская емкость $\left.C_{2, \alpha /(\alpha-1)}\right)$, а также как класс устранимых особенностей для уравнения $\Delta u=u^{\alpha}$. При этом понятие устранимой особенности модифицируется следующим образом. Вместо ограниченных гармонических функций нужно рассматривать неотрицательные решения уравнения $\Delta u=u^{\alpha}$ в области $U \backslash \Gamma$, не обязательно ограниченные. Аналогичный результат справедлив и для множеств на границе области, имеющих подходящую бесселевскую емкость (это утверждение для броуновского движения много проще - множество на границе области полярно, если оно имеет меру нуль).

Как уже упоминалось, неотрицательная гармоническая функция в гладкой области $D$ может быть представлена в виде интеграла $h(x)=\int_{\partial D} k(x, y) \mu(d u)$, где $k(x, y)-$ ядро Пуассона в области $D$, а $\mu(d u)$ - конечная мера на границе области, в определенном смысле служащая слабым пределом решения на границе области. Аналогам этого классического результата для нелинейного уравнения $\Delta u=u^{\alpha}$ посвящен большой цикл работ Е. Б. Дынкина, частично совместных с С. Е. Кузнецовым, подытоженный в двух монографиях [52], [53].

Класс решений нелинейного уравнения обладает рядом свойств, не имеющих аналогов в классе гармонических функций. Так, например, существуют решения, обращающиеся в бесконечность на существенной части границы и даже на всей границе

11 Часть этих результатов, например характеризация полярных множеств для супердиффузий, применима к более общим супердиффузиям.

${ }^{12}$ Работы Ле Галля посвящены так называемой броуновской змее - случайному процессу со значениями в пространстве траекторий. Как и суперпроцесс, броуновскую змею можно рассматривать как предел ветвящейся системы частиц. Змея имеет ряд преимуществ по сравнению с суперпроцессами, но ограничивает класс возможных функций $\psi(u)$. Фактически, естественной для броуновской змеи является лишь функция $\psi(u)=u^{2}$. 
(так называемое максимальное решение, которое доминирует все остальные решения). Стартовой точкой здесь послужила еще одна работа Ле Галля [82], в которой было установлено взаимно однозначное соответствие между положительными решениями уравнения $\Delta u=u^{2}$ в ограниченной гладкой плоской области и их следом на границе - парой $(\Gamma, \nu)$, где замкнутое множество $\Gamma \subset \partial D$ - это множество, вблизи которого решение быстро стремится к бесконечности, а $\sigma$-конечная мера $\nu$ на дополнении $\partial D \backslash \Gamma$ является граничным значением решения в том же смысле, в каком это справедливо для гармонических функций. Плоская область появилась здесь отнюдь не случайно - в этом случае полярных множеств на границе области не существует (в размерности три и больше они уже появляются).

Общий ответ на этот вопрос [52], [53] связан с понятиями умеренных и $\sigma$-умеренных решений. Решение $u$ называется умеренным, если оно мажорируется гармонической функцией. Для умеренных решений граничное значение - это конечная мера, не заряжающая полярных множеств. Решение $u$ называется $\sigma$-умеренным, если оно представимо в виде монотонного вверх предела умеренных решений (такое представление, конечно же, не единственно). Е. Б. Дынкин совместно с С. Е. Кузнецовым [49] доказал, что $\sigma$-умеренные решения могут быть описаны своим тонким следом на границе - парой $(\Gamma, \nu)$, где множество $\Gamma \subset \partial D$ замкнуто в связанной с уравнением, так называемой тонкой топологии, а $\sigma$-конечная мера $\nu$ на дополнении $\partial D \backslash \Gamma$ не заряжает полярных множеств ${ }^{13}$. Более того, соответствующее решение может быть выражено в терминах соответствующего суперброуновского движения. И, наконец, при определенных условиях можно утверждать, что все неотрицательные решения являются $\sigma$-умеренными и, стало быть, все положительные решения однозначно описываются своим тонким следом. Последний результат был сначала получен Б. Мселати [88] для уравнения $\Delta u=u^{2}$ в произвольной размерности, и затем Е. Б. Дынкиным [53] для уравнения $\Delta u=u^{\alpha}, 1<\alpha \leqslant 2$, опять-таки в произвольной размерности. Эквивалентные аналитические результаты, справедливые в том числе и для $\alpha>2$, были получены М. Маркусом и Л. Вероном [83] значительно позднее.

2.8.3. Прочие работы. Упомянем в заключение несколько работ по суперпроцессам, оказавшихся несколько в стороне от направления главного удара. В работе [50] (совместной с С. Е. Кузнецовым) изучаются суперпроцессы, отвечающие уравнению $L u=u^{\alpha}-c u$, где $L-$ эллиптический оператор второго порядка. Авторов интересует существование нетривиального решения этого уравнения с нулевыми граничными условиями. Оказывается, что подобное решение в области $D$ существует тогда и только тогда, когда суперпроцесс в области $D$ с положительной вероятностью не вырождается. Подобный эффект возможен, лишь если $c>0$. Цикл работ [54]-[56] посвящен эксцессивным функциям и границе Мартина для суперпроцессов. В работах [47], [48] описываются линейные аддитивные функционалы от суперпроцессов.

\section{Э. Б. Винберг, С.Е. Кузнецов}

\section{Цитированные работы Е. Б. Дынкина}

[1] "О характеристических числах стохастической матрицы”, Докл. АН $C C C P$, 49:3 (1945), 159-162 (совм. с Н. Дмитриевым).

[2] "Классификация простых групп Ли”, Матем. сб., 18(60):3 (1946), 347-352; англ. пер.: "Classification of simple Lie groups", Selected papers of E.B. Dynkin with

${ }^{13}$ Если $\alpha \geqslant(d+1) /(d-1)$, где $d$ - размерность пространства, то полярных множеств на границе не существует и тонкий след совпадает со следом Ле Галля или, вернее, с его аналогом для $\alpha \neq 2$. В общем случае такой аналог, так называемый грубый след, можно построить, но он не определяет решение однозначно - существуют различные решения, имеющие один и тот же грубый след. 
commentary, eds. A. A. Yushkevich, G. M. Seitz, A. L. Onishchik, Amer. Math. Soc., Providence, RI; International Press, Cambridge, MA, 2000, 23-30.

[3] "О представлении ряда $\log \left(e^{x} e^{y}\right)$ от некоммутирующих $x$ и $y$ через коммутаторы", Матем. сб., 25(67):1 (1949), 155-162.

[4] "Нормированные алгебры Ли и аналитические группы", УМH, 5:1(35) (1950), 135-186; англ. пер.: "Normed Lie algebras and analytic groups", Amer. Math. Soc. Transl., 97, Amer. Math. Soc., Providence, RI, 1953, 66 pp.

[5] “Автоморфизмы полупростых алгебр Ли”, Докл. АН СССР, 76:5 (1951), 629-632.

[6] "Необходимые и достаточные статистики для семейства распределений вероятностей", УМН, 6:1(41) (1951), 68-90; англ. пер.: "Necessary and sufficient statistics for a family of probabiity distributions", Select. Transl. Math. Statist. Probab., 1, Amer. Math. Soc., Providence, RI, 1961, 17-40.

[7] "Максимальные подгруппы классических групп", Тр. ММО, 1, ГИТТЛ, М.-Л., 1952, 39-166; англ. пер.: "Maximal subgroups of the classical groups", Amer. Math. Soc. Transl. (2), 6, Amer. Math. Soc., Providence, RI, 1957, 245-378.

[8] "Полупростые подалгебры полупростых алгебр Ли", Матем. сб., 30(72):2 (1952), 349-462; англ. пер.: "Semisimple subalgebras of semisimple Lie algebras", Amer. Math. Soc. Transl. (2), 6, Amer. Math. Soc., Providence, RI, 1957, 111-243.

[9] "Критерии непрерывности и отсутствия разрывов второго рода для траекторий марковского случайного процесса", Изв. АН СССР. Сер. матем., 16:6 (1952), $563-572$.

[10] "Гомологии компактных групп Ли”, УМН, 8:5(57) (1953), 73-120; англ. пер.: "Homologies of compact Lie groups", Amer. Math. Soc. Transl. (2), 12, Amer. Math. Soc., Providence, RI, 1959, 251-300.

[11] “Топологические характеристики гомоморфизмов компактных групп Ли”, $M a$ тем. сб., 35(77):1 (1954), 129-173; англ. пер.: "Topological characteristics of homomorphisms of compact Lie groups", Amer. Math. Soc. Transl. (2), 12, Amer. Math. Soc., Providence, RI, 1959, 301-342.

[12] "Некоторые предельные теоремы для сумм независимых случайных величин с бесконечными математическими ожиданиями", Изв. АН СССР. Сер. матем., 19:4 (1955), 247-266; англ. пер.: "Some limit theorems for sums of independent random variables with infinite mathematical expectations", Select. Transl. Math. Statist. Probab., 1, Amer. Math. Soc., Providence, RI, 1961, 171-189.

[13] "Строго марковские процессы", Теория вероятн. и ее примен., 1:1 (1956), 149-155 (совм. с А. А. Юшкевичем); англ. пер.: "Strong Markov processes", Theory Probab. Appl., 1:1 (1956), 134-139 (with A. A. Yushkevich).

[14] Основания теории марковских процессов, Физматгиз, М., 1959, 227 с.; англ. пер.: Theory of Markov processes, Pergamon Press, Oxford-London-New York-Paris, 1961, 210 рр.; нем. пер.: Die Grundlagen der Theorie der Markoffschen Prozesse, Grundlehren Math. Wiss., 108, Springer-Verlag, Berlin-Göttingen-Heidelberg, 1961, xi+174 pp.; фр. пер.: Théorie des processus Markoviens, Collection universitaire de mathématiques, 11, Dunod, Paris, 1963, 214 pp.

[15] "Марковские процессы и связанные с ними задачи анализа", УМH, 15:2(92) (1960), 3-24; англ. пер.: "Markov processes and related problems of analysis", Russian Math. Surveys, 15:2 (1960), 1-21; венгер. пер.: Magyar Tud. Akad. Mat. Fiz. Oszt. Kozl., 17 (1967), 231-251.

[16] "Случайное блуждание на группах с конечным числом образующих", Докл. АH CCCP, 137:5 (1961), 1042-1045 (совм. с М. Б. Малютовым); англ. пер.: "Random walk on groups with a finite number of generators", Soviet Math. Dokl., 2 (1961), 399-402 (with M. B. Malyutov); Selected papers of E. B. Dynkin with commentary, eds. A. A. Yushkevich, G. M. Seitz, A.L. Onishchik, Amer. Math. Soc., Providence, RI; International Press, Cambridge, MA, 2000, 481-484. 
[17] Марковские прочессы, Физматгиз, М., 1963, 859 с.; англ. пер.: Markov processes, v. I, II, Grundlehren Math. Wiss., 121, 122, Springer-Verlag, Berlin-Göttingen-Heidelberg, 1965, xii+365 pp., viii+274 pp.

[18] "Марковские процессы и задачи анализа", Proc. Internat. Congr. Mathematicians (Stockholm, 1962), Inst. Mittag-Leffler, Djursholm, 1963, 36-58; англ. пер.: "Markov processes and problems in analysis", Amer. Math. Soc. Transl. (2), 31, Amer. Math. Soc., Providence, RI, 1963, 1-24.

[19] "Оптимальный выбор момента остановки марковского процесса", Докл. АН CCCP, 150:2 (1963), 238-240; англ. пер.: "The optimum choice of the instant for stopping a Markov process", Soviet Math. Dokl., 4 (1963), 627-629; Selected papers of E. B. Dynkin with commentary, eds. A. A. Yushkevich, G. M. Seitz, A. L. Onishchik, Amer. Math. Soc., Providence, RI; International Press, Cambridge, MA, 2000, $485-488$.

[20] "Границы Мартина и неотрицательные решения краевой задачи с наклонной производной”, УМH, 19:5(119) (1964), 3-50; “Письмо в редакцию”, 19:6(120) (1964), 255-256; англ. пер.: "Martin boundaries and non-negative solutions of a boundary value problem with a directional derivative", Russian Math. Surveys, 19:5 (1964), $1-48$.

[21] "Броуновское движение в некоторых симметрических пространствах и неотрицательные собственные функции оператора Лапласа-Бельтрами", Изв. АН CCCP. Cер. матем., 30:2 (1966), 455-478; англ. пер.: "Brownian motion in certain symmetric spaces and nonnegative eigenfunctions of the Laplace-Beltrami operator", Amer. Math. Soc. Transl. (2), 72, Amer. Math. Soc., Providence, RI, 1968, 203-228.

[22] "Общие дополнительные условия для краевой задачи с наклонной производной", Докл. АН СССР, 168:4 (1966), 737-739; англ. пер.: "General supplementary conditions for boundary value problems with oblique derivative", Soviet Math. Dokl., 7:4 (1966), 712-714.

[23] "Общие граничные условия для марковских процессов со счетным множеством состояний”, Теория вероятн. и ее примен., 12:2 (1967), 222-257; "Письмо в редакцию", 13:2 (1968), 379; англ. пер.: "General boundary conditions for denumerable Markov processes", Theory Probab. Appl., 12:2 (1967), 187-221.

[24] “Диффузия тензоров”, Докл. АН CCCP, 6 (1968), 1264-1267; англ. пер.: "Diffusion of tensors", Soviet Math. Dokl., 9 (1968), 532-535.

[25] "О началах странствий марковского процесса", Теория вероятн. и ее примен., 13:3 (1968), 490-493 (совм. с А. А. Юшкевичем); англ. пер.: "On the starting points of incursions of Markov processes", Theory Probab. Appl., 13:3 (1968), 468-470 (with A. A. Yushkevich).

[26] "О продолжениях марковского процесса", Теория вероятн. и ее примен., 13:4 (1968), 708-713; англ. пер.: "On extensions of a Markov process", Theory Probab. Appl., 13:4 (1968), 672-676.

[27] “Граничная теория марковских процессов (дискретный случай)", УМН, 24:2(146) (1969), 3-42; англ. пер.: "Boundary theory for Markov processes. (The discrete case)", Russian Math. Surveys, 24:2 (1969), 1-42.

[28] "Пространство выходов марковского процесса", УМH, 24:4(148) (1969), 89-152; англ. пер.: "The space of exits of a Markov process", Russian Math. Surveys, 24:4 (1969), 89-157.

[29] "Игровой вариант задачи об оптимальной остановке", Докл. АН CCCP, 185:1 (1969), 16-19; англ. пер.: "Game variant of a problem on optimal stopping", Soviet Math. Dokl., 10 (1969), 270-274.

[30] "Начальное и финальное поведение траекторий марковских процессов", УМH, 26:4(160) (1971), 153-172; англ. пер.: "The initial and final behaviour of the trajectories of Markov processes", Russian Math. Surveys, 26:4 (1971), 165-185. 
[31] "Странствия марковского процесса", Теория вероятн. и ее примен., 16:3 (1971), 409-436; англ. пер.: "Wanderings of a Markov process", Theory Probab. Appl., 16:3 (1971), 401-428.

[32] “Интегральное представление эксцессивных мер и эксцессивных функций”, УМН, 27:1(163) (1972), 43-80; англ. пер.: "Integral representation of excessive measures and excessive functions", Russian Math. Surveys, 27:1 (1972), 43-84.

[33] "Регулярные марковские процессы", УМH, 28:2(170) (1973), 35-64; англ. пер.: "Regular Markov processes", Russian Math. Surveys, 28:2 (1973), 33-64.

[34] "Марковские представления стохастических систем", УМН, 30:1(181) (1975), 61-99; англ. пер.: "Markov representations of stochastic systems", Russian Math. Surveys, 30:1 (1975), 65-104.

[35] Управляемые марковские прочессы и их приложения, Наука, М., 1975, 338 с. (совм. с А. А. Юшкевичем); англ. пер.: Controlled Markov processes, Grundlehren Math. Wiss., 235, Springer-Verlag, Berlin-New York, 1979, xvii+289 pp. (with A. A. Yushkevich).

[36] "Sufficient statistics and extreme points", Ann. Probab., 6:5 (1978), 705-730.

[37] "Markov processes and random fields", Bull. Amer. Math. Soc. (N.S.), 3:3 (1980), 975-999.

[38] "Markov processes, random fields and Dirichlet spaces", Phys. Rep., 77:3 (1981), 239-247.

[39] "Green's and Dirichlet spaces associated with fine Markov processes", J. Funct. Anal., 47:3 (1982), 381-418.

[40] "Markov processes as a tool in field theory", J. Funct. Anal., 50:2 (1983), 167-187.

[41] "Gaussian and non-Gaussian random fields associated with Markov processes", J. Funct. Anal., 55:3 (1984), 344-376.

[42] "Random fields associated with multiple points of the Brownian motion", J. Funct. Anal., 62:3 (1985), 397-434.

[43] "Self-intersection gauge for random walks and for Brownian motion", Ann. Probab., 16:1 (1988), 1-57.

[44] An introduction to branching measure-valued processes, CRM Monogr. Ser., 6, Amer. Math. Soc., Providence, RI, 1994, x+134 pp.

[45] "Branching measure-valued processes", Probab. Theory Related Fields, 99:1 (1994), 55-96 (with S. E. Kuznetsov, A. V. Skorokhod).

[46] "Superdiffusions and removable singularities for quasilinear partial differential equations", Comm. Pure Appl. Math., 49:2 (1996), 125-176 (with S. E. Kuznetsov).

[47] "Linear additive functionals of superdiffusions and related nonlinear P.D.E.", Trans. Amer. Math. Soc., 348:5 (1996), 1959-1987 (with S. E. Kuznetsov).

[48] "Natural linear additive functionals of superprocesses", Ann. Probab., 25:2 (1997), 640-661 (with S. E. Kuznetsov).

[49] "Fine topology and fine trace on the boundary associated with a class of semilinear differential equations", Comm. Pure Appl. Math., 51:8 (1998), 897-936 (with S. E. Kuznetsov).

[50] "Extinction of superdiffusions and semilinear PDEs", J. Funct. Anal., 162:2 (1999), 346-378 (with S. E. Kuznetsov).

[51] Selected papers of E. B. Dynkin with commentary, eds. A. A. Yushkevich, G. M. Seitz, A. L. Onishchik, Amer. Math. Soc., Providence, RI; International Press, Cambridge, MA, 2000, xxvi+798 pp.

[52] Diffusions, superdiffusions and partial differential equations, Amer. Math. Soc. Colloq. Publ., 50, Amer. Math. Soc., Providence, RI, 2002, xii+236 pp.

[53] Superdiffusions and positive solutions of nonlinear partial differential equations, Univ. Lecture Ser., 34, Amer. Math. Soc., Providence, RI, 2004, vi+120 pp. 
[54] "Harmonic functions and exit boundary of superdiffusion", J. Funct. Anal., 206:1 (2004), 33-68.

[55] "On extreme X-harmonic functions", Math. Res. Lett., 13:1 (2006), 59-69.

[56] "A note on X-harmonic functions", Illinois J. Math., 50:1-4 (2006), 385-394.

[57] "Enhanced Dynkin diagrams and Weyl orbits", Transform. Groups, 15:4 (2010), 813-841 (with A. N. Minchenko).

\section{Цитированные работы других авторов}

[58] Е. М. Андреев, Э. Б. Винберг, А. Г. Элашвили, "Орбиты наибольшей размерности полупростых линейных групп Ли”, Функи. анализ и его прил., 1:4 (1967), 3-7; англ. пер.: E. M. Andreev, É. B. Vinberg, A. G. Élashvili, "Orbits of greatest dimension of semisimple linear Lie groups", Funct. Anal. Appl., 1:4 (1967), 257-261.

[59] A. Beurling, J. Deny, "Dirichlet spaces", Proc. Nat. Acad. Sci. U.S.A., 45 (1959), 208-215.

[60] R. M. Blumenthal, "An extended Markov property", Trans. Amer. Math. Soc., 85 (1957), 52-72.

[61] A. Borel, J. de Siebenthal, "Les sous-groupes fermés de rang maximum des groupes de Lie clos", Comment. Math. Helv., 23 (1949), 200-221.

[62] H. Cartan, "Théorie générale du balayage en potentiel newtonien", Ann. Univ. Grenoble. Sect. Sci. Math. Phys. (N.S.), 22 (1946), 221-280.

[63] K. L. Chung, "Some new developments in Markov chains", Trans. Amer. Math. Soc., 81 (1956), 195-210.

[64] D. A. Dawson, "Stochastic evolution equations and related measure processes", J. Multivariate Anal., 5 (1975), 1-52.

[65] J.L. Doob, "Markoff chains - denumerable case", Trans. Amer. Math. Soc., 58:3 (1945), 455-473.

[66] J. L. Doob, "Semimartingales and harmonic functions", Trans. Amer. Math. Soc., 77 (1954), 86-121.

[67] А.Г. Элашвили, "Канонический вид и стационарные подалгебры точек общего положения для простых линейных групп Ли", Функи. анализ и его прил., 6:1 (1972), 51-62; англ. пер.: A. G. Élashvili, "Canonical form and stationary subalgebras of points in general position for simple linear Lie groups", Funct. Anal. Appl., 6:1 (1972), 44-53.

[68] I. V. Evstigneev, "Dynkin's work in mathematical economics", Selected papers of E. B. Dynkin with commentary, Amer. Math. Soc., Providence, RI; International Press, Cambridge, MA, 2000, 793-796.

[69] W. Feller, "The parabolic differential equations and the associated semi-groups of transformations", Ann. of Math. (2), 55:3 (1952), 468-519.

[70] W. Feller, "On boundaries and lateral conditions for the Kolmogorov differential equations", Ann. of Math. (2), 65:3 (1957), 527-570.

[71] M. Fukushima, Y. Oshima, M. Takeda, Dirichlet forms and symmetric Markov processes, de Gruyter Stud. Math., 19, Walter de Gruyter \& Co., Berlin, 1994, x+392 pp.

[72] G. A. Hunt, "Some theorems concerning Brownian motion", Trans. Amer. Math. Soc., 81:2 (1956), 294-319.

[73] Дж. А. Хант, Марковские прочессы и потенииаль, Математика. Сб. пер., ИЛ, М., 1962, 283 с.; пер. с англ.: G. A. Hunt, "Markoff processes and potentials. I, II, III", Illinois J. Math., 1, 2 (1957, 1958), 44-93, 316-369, 151-213. 
[74] K. Ito, "The Brownian motion and tensor fields on Riemannian manifold", Proc. Internat. Congr. Mathematicians (Stockholm, 1962), Inst. Mittag-Leffler, Djursholm, $1963,536-539$.

[75] N. Jacobson, "Completely reducible Lie algebras of linear transformations", Proc. Amer. Math. Soc., 2 (1951), 105-113.

[76] Ф.И. Карпелевич, “О неполупростых максимальных подалгебрах полупростых алгебр Ли", Докл. АН СССР, 76:6 (1951), 775-778.

[77] J. R. Kinney, "Continuity properties of sample functions of Markov processes", Trans. Amer. Math. Soc., 74:2 (1953), 280-302.

[78] B. Kostant, "The principal three-dimensional subgroup and the Betti numbers of a complex simple Lie group", Amer. J. Math., 81:4 (1959), 973-1032.

[79] С.Е. Кузнецов, "Построение марковских процессов со случайными моментами рождения и гибели", Теория вероятн. и ее примен., 18:3 (1973), 596-600; англ. пер.: S. E. Kuznetsov, "Construction of Markov processes with random birth and death times", Theory Probab. Appl., 18:3 (1974), 571-575.

[80] С. Е. Кузнецов, "О разложении эксцессивных функций”, Докл. АН СCCP, 214 (1974), 276-278; англ. пер.: S. E. Kuznetsov, "On the decomposition of excessive functions", Soviet Math. Dokl., 15 (1974), 121-124.

[81] J.-F. Le Gall, "The Brownian snake and solutions of $\Delta u=u^{2}$ in a domain", Probab. Theory Related Fields, 102:3 (1995), 393-432.

[82] J.-F. Le Gall, "A probabilistic Poisson representation for positive solutions of $\Delta u=u^{2}$ in a planar domain", Comm. Pure Appl. Math., 50:1 (1997), 69-103.

[83] M. Marcus, L. Véron, "The precise boundary trace of positive solutions of the equation $\Delta u=u^{q}$ in the supercritical case", Perspectives in nonlinear partial differential equations, Contemp. Math., 446, Amer. Math. Soc., Providence, RI, 2007, 345-383.

[84] P. A. Meyer, "Dynkin and the theory of Markov processes", Selected papers of E. B. Dynkin with commentary, Amer. Math. Soc., Providence, RI; International Press, Cambridge, MA, 2000, 763-772.

[85] А.Н. Минченко, "Полупростые подалгебры особых алгебр Ли", Тр. ММО, 67, УРСC, М., 2006, 256-293; англ. пер.: A. N. Minchenko, "The semisimple subalgebras of exceptional Lie algebras", Trans. Moscow Math. Soc., 67, Amer. Math. Soc., Providence, RI, 2006, 225-259.

[86] В. В. Морозов, "О нильпотентном элементе в полупростой алгебре Ли", Докл. АН CCCP, 36:3 (1942), 91-94.

[87] В.В. Морозов, О неполупростых максимальных подгруппах простых групп, Докт. дисс., Казанский ун-т, Казань, 1943, 105 с.

[88] B. Mselati, Classification and probabilistic representation of the positive solutions of a semilinear elliptic equation, Mem. Amer. Math. Soc., 164, № 798, Amer. Math. Soc., Providence, RI, 2004, xvi+121 pp.

[89] D. I. Panyushev, E. B. Vinberg, "The work of Vladimir Morozov on Lie algebras", Transform. Groups, 15:4 (2010), 1001-1013.

[90] D. Ray, "Stationary Markov processes with continuous paths", Trans. Amer. Math. Soc., 82:2 (1956), 452-492.

[91] И. З. Розенкноп, "Некоторые вопросы и приложения гомологической теории полиномиальных идеалов", Тр. ММО, 13, Изд-во Моск. ун-та, М., 1965, 246-323; англ. пер.: I. Z. Rozenknop, "Some problems and applications of the homological theory of polynomial ideals", Trans. Moscow Math. Soc., 13, Amer. Math. Soc., Providence, RI, 1967, 273-358.

[92] Б. Саймон, Модель $P(\varphi)_{2}$ евклидовой квантовой теории поля, Мир, М., 1976, 352 с.; пер. с англ.: B. Simon, The $P(\varphi)_{2}$ Euclidean (quantum) field theory, Princeton Series in Physics, Princeton Univ. Press, Princeton, NJ, 1974, xx+392 pp. 
[93] K. Symanzik, "Euclidean quantum field theory", Local quantum theory, Academic Press, New York-London, 1969, 152-226.

[94] А.Д. Вентцель, "О граничных условиях для многомерных диффузионных процессов", Теория вероятн. и ее примен., 4:2 (1959), 172-185; англ. пер.: A.D. Venttsel', "On boundary conditions for multidimensional diffusion processes", Theory Probab. Appl., 4:2 (1959), 164-177.

[95] А.М. Вершик, "Задача о центральных мерах на пространствах путей градуированных графов", Функи. анализ и его прил., 48:4 (2014), 26-46; англ. пер.: A. M. Vershik, "The problem of describing central measures on the path spaces of graded graphs", Funct. Anal. Appl., 48:4 (2014), 256-271.

[96] А. М. Вершик, А. В. Малютин, "Фазовый переход в задаче о границе-выход для случайных блужданий на группах", Функи. анализ и его прил., 49:2 (2015), 7-20; англ. пер.: А. M. Vershik, A.V. Malyutin, "Phase transition in the exit boundary problem for random walks on groups", Funct. Anal. Appl., 49:2 (2015), 86-96.

[97] Э. Б. Винберг, "Классификация однородных нильпотентных элементов полупростой градуированной алгебры Ли", Тр. сем. по вект. и тенз. анализу, 19 (1979), 155-177; англ. пер.: Е. В. Vinberg, "Classification of homogeneous nilpotent elements of a semisimple graded Lie algebra", Selecta Math. Soviet., 6 (1987), 15-35.

[98] Э. Б. Винберг, А. Г. Элашвили, "Классификация тривекторов девятимерного пространства", Тр. сем. по вект. и тенз. анализу, 18 (1978), 197-233; англ. пер.: È. B. Vinberg, A. G. Èlashvili, "Classification of trivectors of a 9-dimensional space", Selecta Math. Soviet., 7:1 (1988), 63-98.

[99] S. Watanabe, "A limit theorem of branching processes and continuous state branching processes", J. Math. Kyoto Univ., 8 (1968), 141-167.

[100] A. A. Yushkevich, "Dynkin and probability theory", Selected papers of E. B. Dynkin with commentary, Amer. Math. Soc., Providence, RI; International Press, Cambridge, MA, 2000, 385-392.

[101] A. A. Yushkevich, "To the history of strong Markov property", Selected papers of E. B. Dynkin with commentary, Amer. Math. Soc., Providence, RI; International Press, Cambridge, MA, 2000, 773-776. 